\title{
Melkersson-Rosenthal syndrome and psoriasis: An association beyond coincidence?
}

Keywords: Granulomatous, Melkersson-Rosenthal, psoriasis

Anahtar Kelimeler: Granülomatoz, Melkersson-Rosenthal, psoriazis

To the editor,

Melkersson-Rosenthal syndrome (MRS) is characterized by the triad of persistent or recurrent orofacial edema, relapsing facial paralysis and fissured tongue. Its most consistent clinical symptom is recurrent orofacial or lip swelling. Onequarter of MRS patients exhibit the classic triad ${ }^{1}$. The classical histopathological finding is noncaseating epithelioid granulomas. However, absence of these does not exclude the diagnosis. MRS, which has an unknown aetiology, affects primarily young adults. To date, considerable amount of cases of MRS coexistent with psoriasis have been described in the literature ${ }^{2-5}$. In the light of the relevant literature and the case presented here, we discuss the possible association between or coincidence of psoriasis and MRS.

A 49-year-old male presented with a 13-year history of progressive and persistent painless swelling of the upper and lower lips. He also gave a history of asymptomatic eruption on his scalp, face, trunk and upper extremities for several months. He had been treated with oral corticosteroids for recurrent lower motor neuron type facial palsy in the preceding four years. The patient was an otherwise healthy man. He did not have any respiratory, gastrointestinal and neurological symptoms. There was no family history of similar complaints and granulomatous disease such as sarcoidosis or Crohn's disease.
On examination, the upper and lower lips and, prominently, the right side of the face were swollen (Figure 1a). Erythematous papulosquamous eruption was noted on his scalp, face, trunk and upper extremities (Figure 2a, 2b). There was fissured tongue on oral examination (Figure 1b). Specimens obtained by incisional biopsy which was performed on the lower lip did not reveal typical granuloma. However, serial sections showed loose granuloma-like histiocyte accumulations (Figure $3 a, 3 b$ ). The findings of routine blood and stool examination were normal. Chest X-ray did not reveal hilar or mediastinal node enlargement. On the basis of clinical findings, the diagnosis of MRS was made. The biopsy specimen from a plaque lesion on the scalp presented a psoriasiform lesion with parakeratosis and neutrophils in parakeratotic scale. Dermis showed dilated capillaries (Figure 3c). Periodic acid-Schiff staining did not show any fungal organism. The histopathological findings were consistent with psoriasis.

Since the diagnosis of MRS is based on typical clinical features, histological evidence is not essential. Noncaseating granulomas in histopathological examination support the diagnosis. The pathologic examination of our patient showed chronic inflammation with focal histiocyte accumulation. He presented with the full triad of the syndrome. The fissured

Address for Correspondence/Yazışma Adresi: Pınar İncel Uysal MD, Ankara Numune Training and Research Hospital, Clinic of Dermatology, Ankara, Turkey Phone: +90 3125085643 E-mail: pinarincel@hotmail.com Received/Geliş Tarihi: 13.05.2016 Accepted/Kabul Tarihi: 29.07.2016 
tongue is usually asymptomatic and requires no treatment. In those patients with MRS, reconstructive surgery may be a treatment option for orofacial edema, but recurrences are common ${ }^{6}$.

Psoriasis is a common chronic disorder in the world with the prevalence of $0.91 \%$ to $2 \%{ }^{7}$. However, in their case series, Halevy et al. ${ }^{2}$ reported that the prevalence of psoriasis among patients diagnosed with MRS was $50 \%$. The full triad of symptoms was observed in $67 \%$ and fissured tongue in $83 \%$ of psoriatic patients. In addition to this, Galus et al. ${ }^{5}$ reported a patient with the full triad in association with psoriasis and orofacial impetiginisation. More recently, a report from Turkey highlighted the association between psoriasis and fissured tongue in

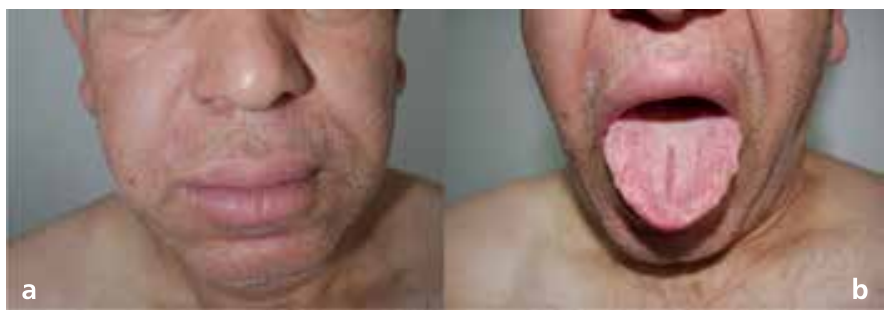

Figure 1. Swelling of the lips and right side of face and psoriatic plaques with squams (a). Fissured tongue (b)

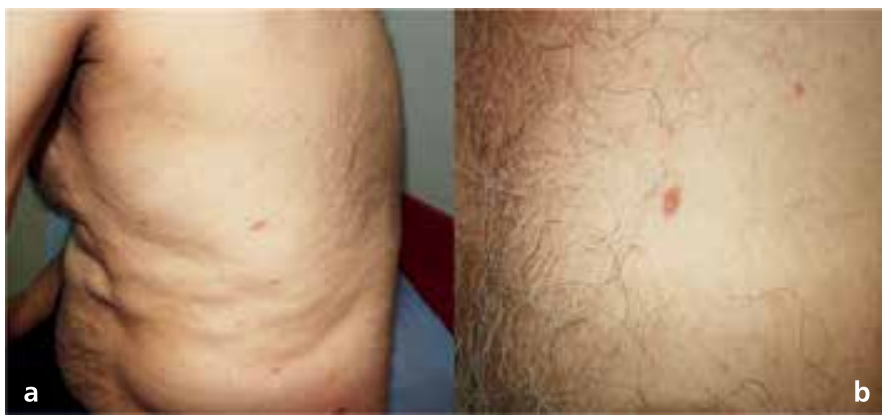

Figure 2. Erythematous plaques and papules on the truncal skin (a). Closer view of a plaque (b)

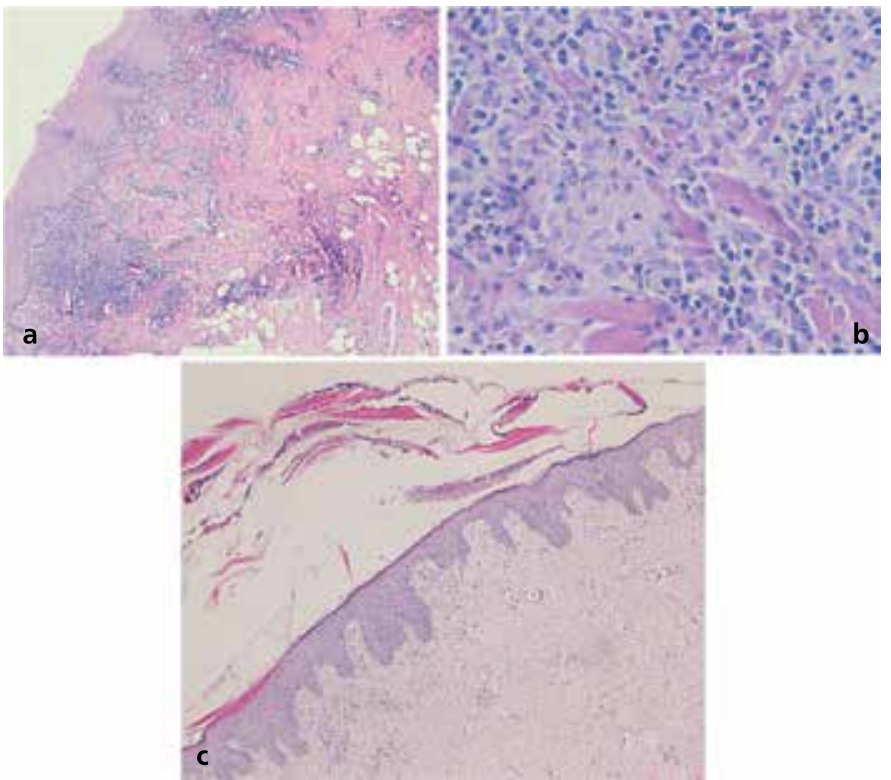

Figure 3. Lip biopsy show chronic inflammation (a). In some areas small granuloma-like histiocyte groups are seen (b). Scalp biopsy shows a psoriasiform lesion (c). Original magnification $(a, c)$ (hematoxylin\&eosin x40), (b) (hematoxylin\&eosin x200) an oligosymptomatic MRS patient ${ }^{4}$. We believe that higher frequency of psoriasis in MRS population may imply an association between MRS and psoriasis rather than coincidence.

In addition, it is well reported that MRS is occasionally a manifestation of Crohn's disease; association of psoriasis with granulomatous disorders such as Crohn's disease and sarcoidosis have been reported in the literature 8,9 . Furthermore, it is well established that both MRS and psoriasis show clinical improvement with the use of tumor necrosis factor-alpha (TNF- $\alpha$ ) blocker. On the other hand, it is also interesting that in their case study, Gaudio et al. 3 reported neurological episodes of MRS after anti-TNF therapy in a patient with psoriatic arthritis. Apart from all these, fissured tongue is another common aspect. As it is well known, estimated incidence of fissured tongue, which is a component of MRS, is $10-15 \%$ in healthy population whereas it is more frequent in psoriatic patients 10 .

The case presented here confirms the hypothesis of association between MRS and psoriasis rather than coincidence. Even though psoriasis is a relatively common disease, it seems that the frequency in MRS group is quite higher than expected. Thus, we suggest that it may be a 'variable' entity of this syndrome. We hope awareness of this coexistence will lead further reports and better understanding of the 'overlooked' relationship between MRS and psoriasis.

\section{Ethics}

Informed Consent: Consent form was filled out by all participants. Peer-review: Internally peer-reviewed.

\section{Authorship Contributions}

Surgical and Medical Practices: Pınar Incel Uysal, Concept: Başak Yalçın, Pınar İncel Uysal, Design: Pınar Incel Uysal, Data Collection or Processing: Pınar İncel Uysal, Başak Yalçın, Analysis or Interpretation: Pınar İncel Uysal, Serra Kayaçetin, Önder Bozdoğan, Literature Search: Pınar İncel Uysal, Writing: Pınar İncel Uysal.

Conflict of Interest: No conflict of interest was declared by the authors. Financial Disclosure: The authors declared that this study received no financial support.

\section{References}

1. Greene RM, Rogers RS: Melkersson-Rosenthal syndrome: a review of 36 patients. J Am Acad Dermatol 1989;21:1263-70.

2. Halevy S, Shalom G, Trattner A, Bodner L: Melkersson-Rosenthal syndrome: a possible association with psoriasis. J Am Acad Dermatol 2012;67:795-6.

3. Gaudio A, Corrado A, Santoro N, et al. Melkersson Rosenthal syndrome in a patient with psoriatic arthritis receiving etanercept. Int I Immunopathol Pharmacol 2013;26:229-33.

4. Elmas OF, Kizlyel O, Metin SM, Aktas A: Merkelsson-Rosenthal syndrome: is psoriasis a new component of the syndrome? J Turgut Ozal Med Cent 2015;22:213-4.

5. Galus R, Borowska K, Jędrych M, Zabielski S: Melkerrson-Rosenthal Syndrome associated with psoriasis vularis and orofacial impetiginization. Our Dermatol Online 2014;5:182-3.

6. Liu R, Yu S: Melkersson-Rosenthal syndrome: a review of seven patients. $J$ Clin Neurosci 2013;20:993-5.

7. Parisi R, Symmons DP, Griffiths $C E$, et al: Global epidemiology of psoriasis: a systematic review of incidence and prevalence. I Invest Dermatol 2013;133:377-85.

8. Christophers E: Comorbidities in psoriasis. Clin Dermatol 2007:25:529-34.

9. Cohen $A D$, Dreiher J, Birkenfeld S: Psoriasis associated with ulcerative colitis and Crohn's disease. J Eur Acad Dermatol Venereol 2009;23:561-5.

10. Daneshpazhooh MS, Moslehi H, Akhyani M, Etesami M: Tongue lesions in psoriasis: a controlled study. BMC Dermatol 2004;4:16. 